\title{
Mie Resonances in Water Spheres for Microwave Metamaterials and Antennas
}

\author{
Jacobsen, Rasmus Elkjær; Arslanagic, Samel; Laurynenka, Andrei
}

Published in:

The Radio Science Bulletin

Publication date:

2020

Document Version

Publisher's PDF, also known as Version of record

Link back to DTU Orbit

Citation (APA):

Jacobsen, R. E. Arslanagic, S., \& Laurynenka, A. (2020). Mie Resonances in Water Spheres for Microwave Metamaterials and Antennas. The Radio Science Bulletin, 1.

\section{General rights}

Copyright and moral rights for the publications made accessible in the public portal are retained by the authors and/or other copyright owners and it is a condition of accessing publications that users recognise and abide by the legal requirements associated with these rights.

- Users may download and print one copy of any publication from the public portal for the purpose of private study or research.

- You may not further distribute the material or use it for any profit-making activity or commercial gain

- You may freely distribute the URL identifying the publication in the public portal

If you believe that this document breaches copyright please contact us providing details, and we will remove access to the work immediately and investigate your claim. 


\title{
Mie Resonances in Water Spheres for Microwave Metamaterials and Antennas
}

\author{
Rasmus E. Jacobsen, Samel Arslanagić, and Andrei V. Lavrinenko
}

\begin{abstract}
All-dielectric metamaterials rely on high-permittivity inclusions, which support Mie resonances. At microwave frequencies, distilled water exhibits a relatively high permittivity with tunable dynamic properties that are interesting for microwave devices, although absorption in water reduces their efficiency. In this paper, we investigate the scattering and absorption of single water spheres of various sizes and temperatures. The study is based on analytical calculations of Mie resonances. We also investigate the balanced electric/magnetic dipole excitation for more directive scattering in the forward direction. Finally, we discuss the major differences and similarities between water spheres and water cylinders. The results can be used as guidelines for metamaterial-based components as well as for dielectric resonator antenna designs.
\end{abstract}

\section{Introduction}

All-dielectric metamaterials (MMs) and metasurfaces (MSs) are preferred over the metallic versions because they possess lower intrinsic losses and potentially higher efficiency [1]. They are typically composed of high-index inclusions in a low-index host, where Mie resonances are excited in each individual inclusion. At optical frequencies, the variety of highindex materials is poor compared to microwave frequencies. We find water of particular interest because it holds the potential to be the fundamental element in cheap and bio-friendly microwave MS wave transformers (such as transmitarrays and reflectarrays), MS absorbers, and dielectric resonator antennas, to mention a few. Several water-based devices have already been demonstrated; see, e.g., [2-8]. The dynamic properties of water's permittivity - along with its liquid stateoffer multiple tuning parameters. However, the absorption in water constitutes a major obstacle toward its application for nonabsorber devices, and this is particularly the case for high frequencies. Thus, water inclusions belong in general to lossy high-permittivity particles in MSs. Nevertheless, losses are to some extent moderate up to about $2 \mathrm{GHz}$ at $20^{\circ} \mathrm{C}$, which is the frequency range of our interest.

The purpose of this work is to study the scattering and absorption efficiencies of a subwavelength resonant

Manuscript received 23 December 2019.

R. E. Jacobsen and A. V. Lavrinenko are with the Department of Photonics Engineering, Technical University of Denmark, Oersteds Plads 345A, 2800 Kongens Lyngby, Denmark; e-mail: rajac@fotonik.dtu.dk and alav@fotonik.dtu.dk.

S. Arslanagic is with the Department of Electrical Engineering, Technical University of Denmark, Oersteds Plads 348, 2800 Kongens Lyngby, Denmark; e-mail: sar@elektro.dtu.dk. water inclusion. Scattering and absorption of microwaves and light in rain (i.e., a collection of small water droplets) were studied many years ago $[9,10]$. We render variations of both temperature and size to provide a more elaborate analysis and deeper insight compared to our previous efforts [11, 12]. Here we focus on the spherical shape because it resembles many of the water inclusions found in previously reported MSs. In addition, the canonical problem can be treated analytically using spherical vector wave formulation, eliminating the need for heavy numerical simulations, and thus enabling more extensive studies. Both electric and magnetic dipoles can be excited in the sphere, but we pay most of our attention to the latter because it is the dominant mode. MSs with dielectric inclusions satisfying the first Kerker condition are currently of great interest [7, 13-15]. We demonstrate that this condition can also be satisfied in a water sphere, and we investigate how it is affected by the water losses.

This letter is organized as follows: section II presents the configuration and quantities as well as the obtained results; a summary and conclusions are given in section III. Throughout the work, the time factor $\exp (j \omega t)$, with the angular frequency $\omega$ and the time $t$, is assumed and suppressed.

\section{Configuration and Results}

We consider the canonical configuration sketched in Figure 1a: a linearly polarized plane wave incident on a sphere of distilled water with radius $r_{\mathrm{w}}$, relative permittivity $\varepsilon_{\mathrm{r}, \mathrm{w}}=\varepsilon_{\mathrm{r}, \mathrm{w}}^{\prime}-j \varepsilon_{\mathrm{r}, \mathrm{w}}^{\prime \prime}$, relative permeability $\mu_{\mathrm{r}, \mathrm{w}}=1$, and temperature $T_{\mathrm{w}}$. The sphere is enclosed by free space with the permittivity and permeability $\varepsilon_{0}$ and $\mu_{0}$, respectively. A rectangular $(x, y, z)$ coordinate system is introduced with the associated spherical $(r, \theta$, $\phi)$ coordinate system. The incident electric field is $\mathrm{x}$ polarized and given by $\mathbf{E}^{\mathrm{i}}=\widehat{\mathbf{x}} E_{0} \exp \left(-j k_{0} z\right)$ with $E_{0}=1 \mathrm{~V} / \mathrm{m}$. The model of the relative water permittivity is taken from [16] and is shown in Figure 1b. The response of the sphere is described by the absorption, scattering, and extinction efficiencies, related by $Q_{\text {abs }}=Q_{\text {ext }}-Q_{\text {sca }}$, where [10, chap. 4]

$$
\begin{aligned}
& Q_{\text {sca }}=\frac{2}{\left(k_{0} r_{\mathrm{w}}\right)^{2}} \sum_{n=1}^{\infty}(2 n+1)\left(\left|a_{n}\right|^{2}+\left|b_{n}\right|^{2}\right) \\
& Q_{\text {ext }}=\frac{2}{\left(k_{0} r_{\mathrm{w}}\right)^{2}} \sum_{n=1}^{\infty}(2 n+1) \operatorname{Re}\left\{a_{n}+b_{n}\right\}
\end{aligned}
$$

where $k_{0}=2 \pi / \lambda_{0}$ is the free-space wavenumber and $\lambda_{0}$ the free-space wavelength. The solutions to the 


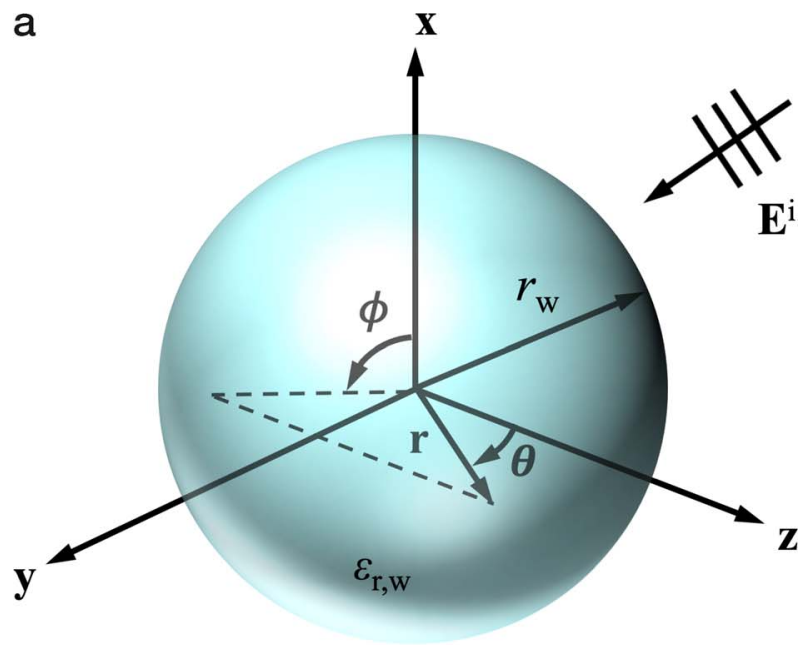

$\varepsilon_{0}, \mu_{0}$

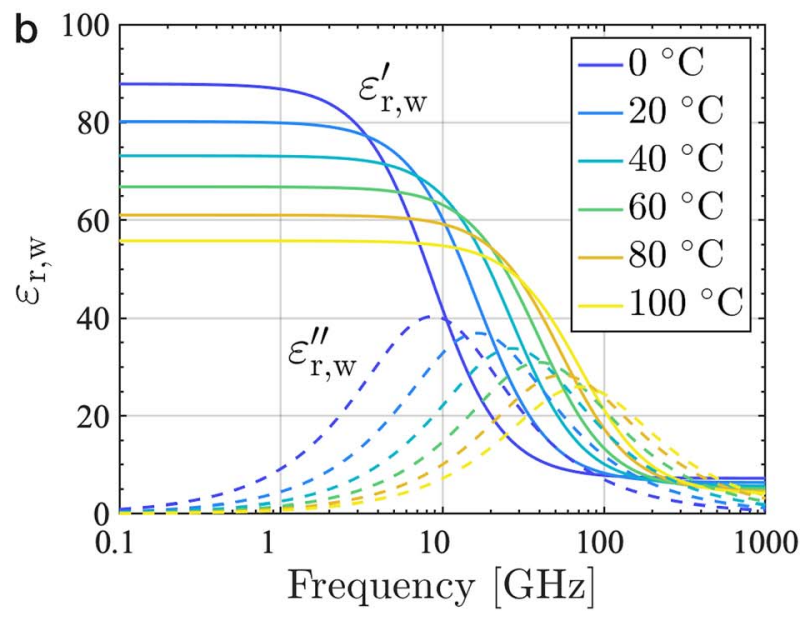

Figure 1. (a) Sketch of the water sphere illuminated by a plane wave and (b) the complex relative permittvity of water $\left(\varepsilon_{\mathrm{r}, \mathrm{W}}=\varepsilon_{\mathrm{r}, \mathrm{W}}^{\prime}-j \varepsilon_{\mathrm{r}, \mathrm{W}}^{\prime \prime}\right)$ as a function of frequency and temperature [13].

expansion coefficients of the $\mathrm{TE}^{r}$ and $\mathrm{TM}^{r}$ scattered field components, $a_{n}$ and $b_{n}$, respectively, can be found in $[10,11]$. Each $n$ corresponds to a different mode excited in the sphere, with $n=1$ being the lowest-order dipole mode.

Both magnetic $\left(a_{1}\right)$ and electric $\left(b_{1}\right)$ dipoles, as well as quadrupoles $(n=2)$, can be excited in the water sphere. However, the largest interaction with the field of the incident plane wave comes with the magnetic dipole as the round shape of the sphere stimulates circulating polarization currents $[2,11]$. At resonance, the magnetic dipole gives rise to a peak in both scattering and absorption. The losses in water reduce the overall efficiency, especially at frequencies above $1 \mathrm{GHz}$, but this also depends on the temperature of water; cf. Figure 1b. The total magnetic and electric fields in the $x z$-plane of a sphere with $r_{\mathrm{w}}=50 \mathrm{~mm}$ at its magnetic dipole resonance frequency $(331 \mathrm{MHz})$ is shown in Figure 2. The electric field is maximal near the periphery inside the water sphere, around which it circulates. This results

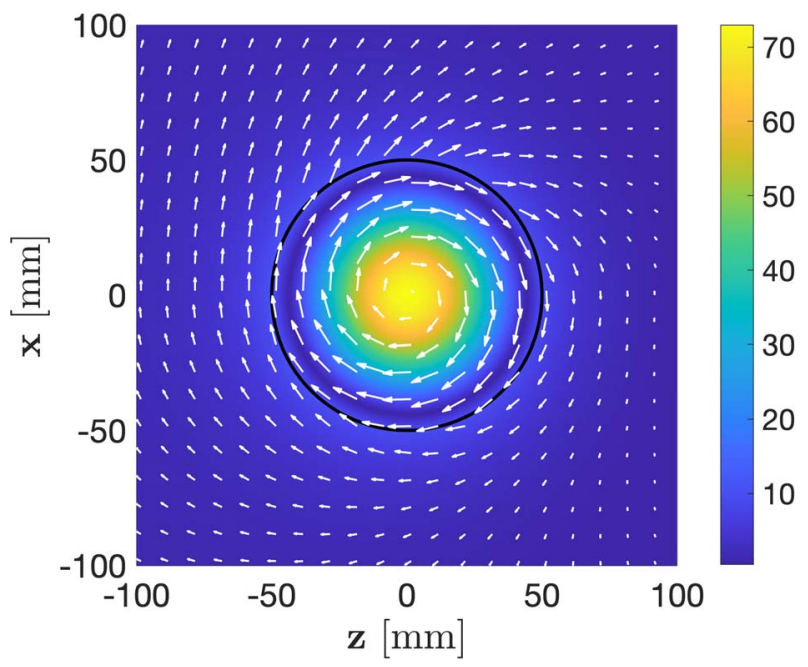

Figure 2. Total magnetic and electric fields in the $x z$-plane for a sphere with $r_{\mathrm{w}}=50 \mathrm{~mm}$ and $T_{\mathrm{w}}=20^{\circ} \mathrm{C}$. The frequency is $331 \mathrm{MHz}$. Colors show the total magnetic field magnitude normalized with the incident magnetic field magnitude. The arrows display the magnitude and direction of the total electric field (linear scale).

in an enhanced magnetic field at the center of the sphere.

As the efficiency of a system composed of resonant water spheres - or any other resonant water structures-depends on both frequency and water temperature, we investigate the performance for single resonant spheres of various sizes and resonance frequencies. We use the single-scattering albedo $Q_{\text {sca }} / Q_{\text {ext }}$ to show the amount of the extinct power that is scattered. The magnetic dipole resonance frequency $f_{\mathrm{r}}$ and the single-scattering albedo (as a percentile) as functions of the sphere radius and water temperature are shown in Figure 3. The calculation is performed for radii (temperatures) between $20 \mathrm{~mm}\left(0^{\circ} \mathrm{C}\right)$ and $200 \mathrm{~mm}$ $\left(100^{\circ} \mathrm{C}\right)$. The resonance frequency increases with a decreasing radius and/or temperature due to the change in the water's permittivity. As a consequence of the higher frequency and/or lower temperature, the efficiency decreases. The $50 \%$ albedo level is shown by the red line in the figures, and we observe that the increase in the resonance frequency dramatically reduces the range of spheres with an albedo level above 50\%. At room temperature $\left(20^{\circ} \mathrm{C}\right)$, the spheres with resonance frequencies higher than $144 \mathrm{MHz}$ exhibit albedo levels lower than $50 \%$, and at $1 \mathrm{GHz}$, the albedo level is reduced to only $13 \%$. The calculated albedo levels are comparable to the albedo levels for systems composed of resonant spherical water elements as well as other types of resonant electrically small water elements [2, 8].

Having considered fundamental properties of dipole resonances in water spheres, we next explore their possibility of providing enhanced scattering in the forward directions because transmitting MSs rely on such elements. This can be achieved with elements satisfying Kerker's first condition, which exhibit 

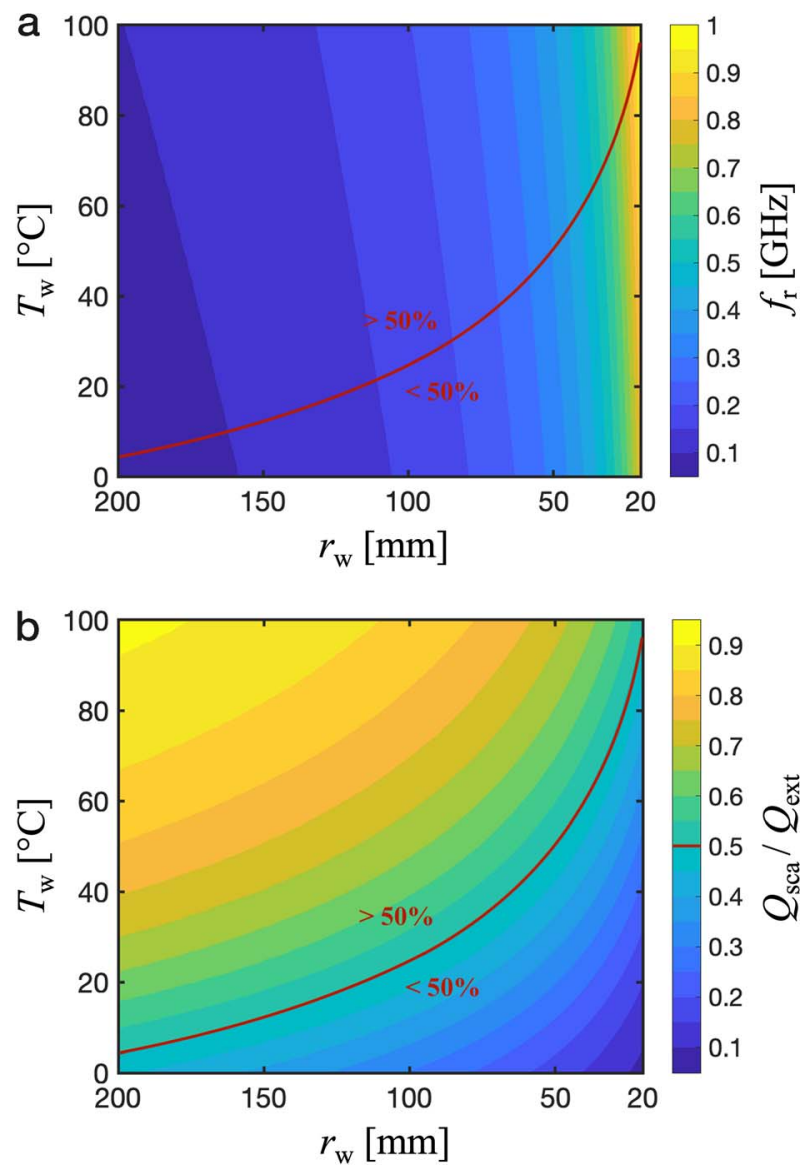

Figure 3. Colors show (a) the magnetic dipole resonance frequency $\left(f_{r}\right)$ and (b) the single-scattering albedo $\left(Q_{\text {sca }} / Q_{\text {ext }}\right)$ as functions of the sphere radius $\left(r_{\mathrm{W}}\right)$ and water temperature $\left(T_{\mathrm{w}}\right)$. The red lines display the $50 \%$ effeciency boundary.

suppressed (enhanced) backward (forward) scattering [15]. For a sphere this occurs when $a_{1} \approx b_{1}$ corresponding to $m=v p$, with $m(p)$ being the induced magnetic (electric) dipole moment and $v$ the speed of the electromagnetic wave in the host medium. This assumes that the dipole modes are the dominant modes in the sphere, and at low frequencies they are dominant. We analyze this condition by evaluating the front-to-back ratio $(\mathrm{FBR})$ :

$$
\mathrm{FBR}=\frac{\sigma_{\mathrm{s}}^{\prime}(0,0)}{\sigma_{\mathrm{s}}^{\prime}(\pi, 0)}=\left|\frac{\sum_{n=1}^{\infty}(2 n+1)\left(a_{n}+b_{n}\right)}{\sum_{n=1}^{\infty}(2 n+1)(-1)^{n}\left(a_{n}-b_{n}\right)}\right|^{2}
$$

where $\sigma_{\mathrm{s}}^{\prime}(\theta, \phi)$ is the differential scattering cross section [10, chap. 4]. From the denominator in Eq. (3), it is obvious that for $a_{1}=b_{1}$ FBR will increase dramatically if we assume that the other modes are weakly contributing to scattering. The maximized FBR, where Kerker's first condition is satisfied, was computed for various sphere sizes at $20^{\circ} \mathrm{C}$. The result is shown in Figure 4 with the frequency included. As the radius increases, the FBR increases significantly because of the

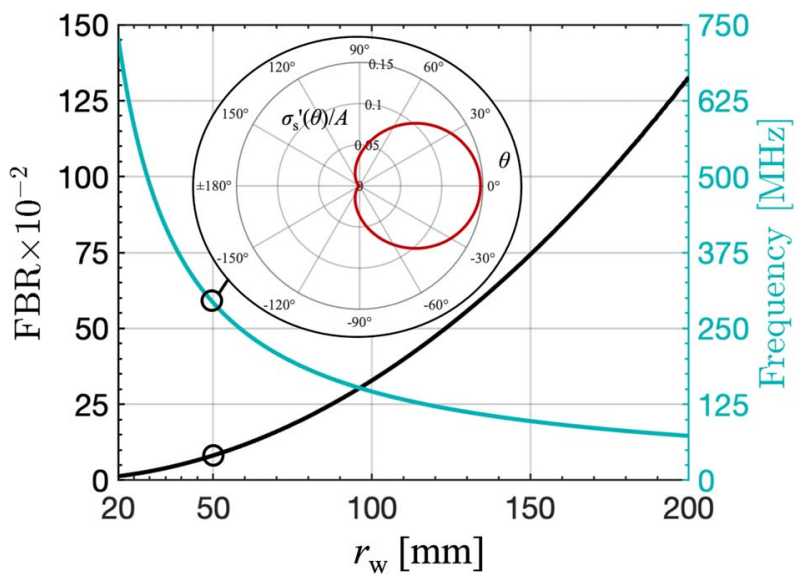

Figure 4. FBR and frequency as functions of the sphere radius $\left(r_{\mathrm{w}}\right)$ at $20^{\circ} \mathrm{C}$ water temperature. The inset shows the normalized differential scattering cross section $\sigma_{s}^{\prime}(\theta) / A$ (with $A=\pi r_{\mathrm{w}}^{2}$ ) in the $x z$-plane for a sphere with $r_{\mathrm{W}}=50 \mathrm{~mm}$.

decrease in frequency and, hence, the decrease of losses in water. This shows that the Kerker condition is highly affected by losses. The normalized differential scattering cross section $\sigma_{\mathrm{s}}^{\prime}(\theta, 0) / A$, with $A=\pi r_{\mathrm{w}}^{2}$ being the cross-sectional area of the sphere, is shown as an inset in Figure 4 for a sphere with $r_{\mathrm{w}}=50 \mathrm{~mm}$. The scattering pattern resembles the characteristic cardioid that two balanced magnetic and electric dipoles exhibit. It should be noted that the total scattering is very low because the dipoles are weakly excited. To maximize the scattering, the dipole resonance frequencies must overlap, but the fulfillment of the condition $a_{1}=b_{1}$ remains. Other larger element shapes satisfying Kerker's first condition exhibit greater scattering, but their sizes are also significantly larger [7, 13-15]. Using elements composed of both metal and water, it can be shown that the FBR can be enhanced as well as the total scattering efficiency. These results will be reported elsewhere.

As a final remark we note that related cylindrical structures were also investigated [12]. Both TE and TM polarizations were studied, with the latter having a dominating monopole mode. The TE polarization response resembles that of the sphere, and, only for this polarization, Kerker's conditions can be satisfied. We calculated the dipole moments and found that the electric dipole moment is highly polarization sensitive, whereas the magnetic dipole moment is identical for both polarizations. The cylindrical structures may prove useful for, e.g., anisotropic MSs.

\section{Summary and Conclusions}

Scattering of plane waves by resonant water spheres of various sizes and temperatures was investigated. The scattering and extinction efficiencies were calculated and compared, and we showed that water spheres at room temperature $\left(20^{\circ} \mathrm{C}\right)$ and with resonance frequencies above $144 \mathrm{MHz}$ have single-scattering albedo levels lower than $50 \%$. The efficiencies of the 
single spheres are similar to those for the systems of spheres reported previously. In addition, the differential scattering cross section was analyzed with a focus on maximizing (minimizing) the forward (backward) scattering, where the water losses also had a negative impact. Finally, we discussed the main difference between water spheres and cylinders. The results can be applied directly or as guidelines for designing metamaterial-based elements of circuitry as well as for constructing water-based dielectric resonator antennas.

\section{References}

1. S. Jahani and Z. Jacob, "All-Dielectric Metamaterials," Nature Nanotechnology, 11, 1, January 2016, pp. 23-36.

2. A. Andryieuski, S. M. Kuznetsova, S. V. Zhukovsky, Y. S. Kivshar, and A. V. Lavrinenko, "Water: Promising Opportunities for Tunable All-Dielectric Electromagnetic Metamaterials," Scientific Reports, 5, August 2015, p. 13535

3. X. Cai, S. Zhao, M. Hu, J. Xiao, N. Zhang, et al., "WaterBased Fluidic Radio Frequency Metamaterials," Journal of Applied Physics, 122, 18, November 2017, p. 184101.

4. R. E. Jacobsen, A. V. Lavrinenko, and S. Arslanagić, "Water-Based Metasurfaces for Effective Switching of Microwaves," IEEE Antennas and Wireless Propagation Letters, 17, 4, February 2018, pp. 571-574.

5. X. Yang, D. Zhang, S. Wu, Y. Yin, L. Li, et al., "Reconfigurable All-Dielectric Metasurface Based on Tunable Chemical Systems in Aqueous Solution," Scientific Reports, 7, June 2017, p. 3190.

6. Y. J. Yoo, S. Ju, S. Y. Park, Y. J. Kim, J. Bong, et al., "Metamaterial Absorber for Electromagnetic Waves in Periodic Water Droplets," Scientific Reports, 5, September 2015, p. 14018.

7. P. J. Bradley, M. O. M. Torrico, C. Brennan, and Y. Hao,
"Printable All-Dielectric Water-Based Absorber," Scientific Reports, 8, September 2018, p. 14490.

8. R. E. Jacobsen, A. V. Lavrinenko, and S. Arslanagić, "Electrically Small Water-Based Hemispherical Dielectric Resonator Antenna," Applied Sciences, 9, 22, November 2019, p. 4848.

9. A. L. Aden, "Microwave Reflection From Water Spheres," American Journal of Physics, 19, 163, 1951, pp. 163-167.

10. C. F. Bohren and D. R. Huffman, Absorption and Scattering of Light by Small Particles, Weinheim, Germany, Wiley-VCH, 1983.

11. R. E. Jacobsen, S. Arslanagić, and A. V. Lavrinenko, "Fundamental Properties of Mie Resonances in Water Spheres," Presented at URSI EM Theory Symposium, EMTS 2019, San Diego, CA, May 27-31, 2019, pp. 1-4.

12. R. E. Jacobsen, S. Arslanagić, and A. V. Lavrinenko, "Fundamental Properties of Mie Resonances in Water Cylinders - TM and TE Case Studies," Presented at URSI EM Theory Symposium, EMTS 2019, San Diego, CA, May 27-31, 2019, pp. 1-4.

13. I. Staude, A. E. Miroshnichenko, M. Decker, N. T. Fofang, S. Liu, et al., "Tailoring Directional Scattering Through Magnetic and Electric Resonances in Subwavelength Silicon Nanodisks," Optical Letters, 7, 9, August 2013, pp. 7824-7832.

14. M. Odit, P. Kapitanova, P. Belov, R. Alaee, C. Rockstuhl, et al., "Experimental Realisation of All-Dielectric Bianisotropic Metasurfaces," Applied Physics Letters, 108, 22, May 2016, p. 221903.

15. D. Tzarouchis and A. Sihvola, "Light Scattering by a Dielectric Sphere: Perspectives on the Mie Resonances," Applied Sciences, 8, 2, January 2018, p. 184.

16. W. Ellison, "Permittivity of Pure Water, at Standard Atmospheric Pressure, Over the Frequency Range 0-25 $\mathrm{THz}$ and the Temperature Range $0-100{ }^{\circ} \mathrm{C}$," Journal of Physics Chemistry Reference Data, 36, 1, March 2007, pp. $1-18$. 